\title{
LOS PRINCIPIOS RECTORES DEL ESPACIO EUROPEO DE EDUCACIÓN SUPE- RIOR VIRTUAL
}

La educación europea superior se encuentra, desde el año 1999, sometida a importantes cambios en el marco de la creación del Espacio Europeo de Educación Superior. A la convergencia de la estructuras y a la implantación del crédito europeo que conforman la estructura formal hay que añadir el cambio en las metodologías de enseñanza y aprendizaje a través de las nuevas tecnologías. Esto supone un nuevo cambio en los roles de docentes y discentes y en los materiales educativos. Este artículo ofrece una revisión de los principios que históricamente han guiado la enseñanza a distancia universitaria y que sientan las bases de los principios que deben regir la implantación de un Espacio Virtual de Educación Superior.

Palabras clave: nuevas tecnologías; educación superior; convergencia europea

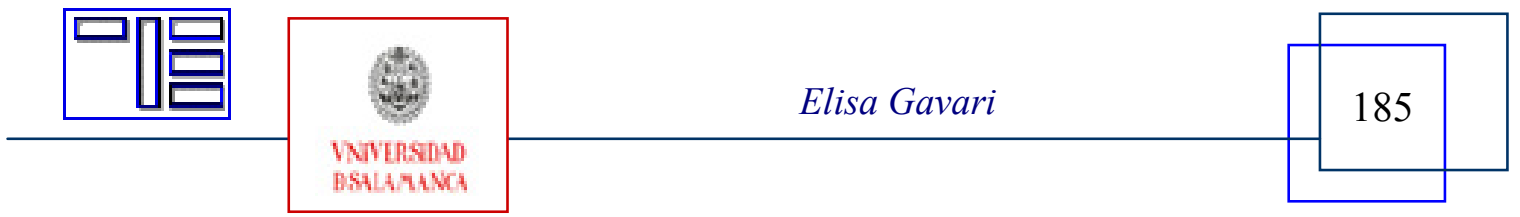


Revista Electrónica Teoría de la Educación.

Educación y Cultura en la Sociedad de la Información.

http://www.usal.es/teoriaeducacion

Vol. 7. N2. Diciembre 2006

\section{GUIDING PRINCIPLES OF THE VIRTUAL EUROPEAN HIGHER EDUCATION SPACE}

European higher education is, since 1999, in a process of relevant changes due to the setting up of the European Higher Education Space. In addition to the convergence of structures and the implementing of the European credit that shape the formal structure we have to add the changes in the teaching and learning methodologies through new technologies. This means changes in the role of teachers and students as well as in the education texts. This article offers a revision of the principles that historically have guided university distance education and that must lead the implementing of a Virtual European Higher Education Space.

Key words: new technologies; higher education; European convergence.

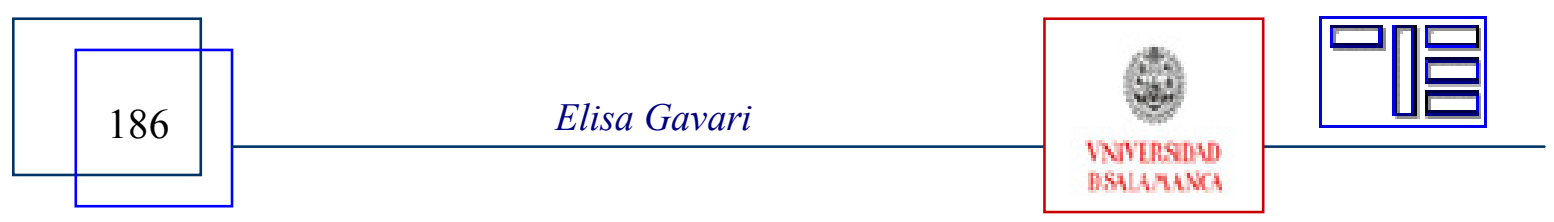




\section{LES PRINCIPES RÉCTEURS DE L'ESPACE D'ÉDUCATION SUPÉRIEUR VIRTUEL}

L'éducation européen supérieure est mise, depuis l'anée 1999, dans un procés marqué par d'importantes changements, dans le cadre de la création de 1'Espace Européen d'Éducation Supérieur. À la convergence des estructures et à l'implantation du crédit européen che conforment la structure formelle il faut ajouter le changement dans les methodologies d'enseignement et apprentisage à travers les nouvelles technologies. Ce changement signifie des nouveaux roles des professeurs et élèves et des nouveles materiaux éducatives. Cet article offer une revision des principes que historiquement ont guide 1'enseignement supérieure ouverte et que constituent les bases de la creation d'un espace d'enseignement virtuel d'éducation supérieure.

Mots clefs: nouveaux technologies; education supérieure; convergence européenne

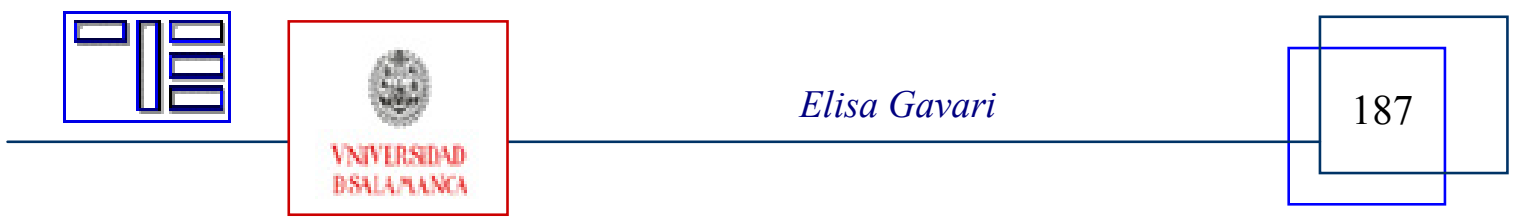


Revista Electrónica Teoría de la Educación.

Educación y Cultura en la Sociedad de la Información.

http://www.usal.es/teoriaeducacion

Vol. 7. No2. Diciembre 2006

\section{LOS PRINCIPIOS RECTORES DEL ESPACIO EUROPEO DE EDUCACIÓN SUPERIOR VIRTUAL}

Elisa Gavari

egavari@edu.uned.es

Universidad Nacional de Educación a Distancia (ESPAÑA)

\section{1.- INTRODUCCIÓN}

Las universidades han ido adaptándose a las necesidades de formación de cada momento histórico. En el último cuarto del siglo XX, la sociedad del conocimiento impone a la enseñanza superior la formación de los ciudadanos para una sociedad marcada por la invasión de las nuevas tecnologías de información y comunicación en todos los espacios. Las universidades europeas además tienen que responder a un reto añadido, como es, cumplir los acuerdos para la creación del Espacio Europeo de Educación Superior caracterizado por la convergencia y la comparabilidad de los sistemas. Esta cooperación entre los Estados también va a adoptarse en la dimensión virtual. En este artículo voy a describir los principios rectores que han guiado la educación superior a distancia desde sus inicios y que son los fundamentos del Espacio Europeo de Educación Superior Virtual.

\section{2.- LA GÉNESIS DEL ESPACIO EUROPEO DE EDUCACIÓN SUPERIOR: LOS CRÉ- DITOS}

Las Comunidades Europeas no consideraron la importancia de definir una educación común europea hasta principios de los setenta. En ese momento se presentaron las primeras propuestas acerca de una educación europea supranacional que no debía ni armonizar ni interferir en la educación que se impartía en los sistemas educativos nacionales, que se consideraban potestad de los Estados. Las autoridades comunitarias utilizaron la extraña expresión "Dimensión europea de la educación" para referirse a las medidas educativas europeas que debían desarrollarse, pero siempre respetando las tradiciones y la diversidad de los sistemas educativos nacionales de los Estados miembros. La Dimensión europea era ciertamente tímida en comparación con otras políticas públicas que

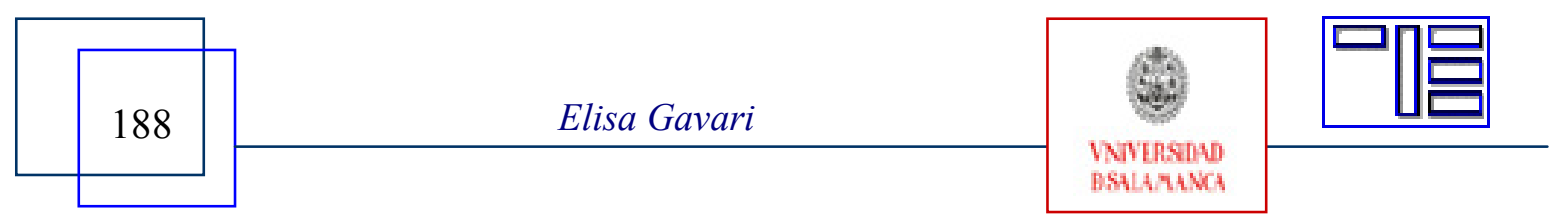


estaban mucho más definidas a nivel supranacional. Las únicas cuestiones en las que ministros de educación europeos consiguieron ponerse de acuerdo fueron las siguientes (Etxeberria, 2000, 21):

- la mejora de la correspondencia entre los sistemas educativos de Europa;

- la recopilación de documentación y estadísticas del sector educativo europeo;

- la intensificación de la cooperación entre los centros de enseñanza superior;

- el reconocimiento de la libertad de circulación y movilidad de profesores, estudiantes e investigadores;

- la mejora de la enseñanza de las lenguas extranjeras;

- y la mejora del reconocimiento académico de los títulos y períodos de estudio.

Estas propuestas se materializaron en el primer Programa de Acción de la Comunidad en Educación que permitió numerosas visitas de estudio e intercambio de docentes y alumnos de primaria y secundaria, y la incorporación de algunos contenidos sobre Europa en los currícula y en las actividades escolares.

En los años ochenta la Comunidad Europea perdió el adjetivo económica en favor de la consecución de objetivos de carácter político. El proyecto de integración de los Estados europeos dio un paso adelante y las teorías acerca de la interdependencia de los Estados soberanos eclipsaron las teorías de la cooperación. Las tesis federalistas fueron cogiendo más adeptos y desembocaron, en 1984, en la presentación del primer proyecto de Tratado de la Unión en el que se proponía, por primera vez, la ciudadanía de la Unión. En este contexto se crea en 1987 el Programa Erasmus cuyo núcleo lo constituye el crédito ECTS. En los orígenes de dicho Programa, el crédito europeo permitía el reconocimiento de períodos en el extranjero lo que permitía aumentar la calidad y el volumen de la movilidad estudiantil en Europa. Recientemente, el ECTS se ha convertido en un sistema de acumulación y transferencia de aprendizajes que permite que los programas de estudio sean fácilmente comparables para todos los estudiantes, en el marco del Espacio Europeo de Educación Superior, independientemente de que sean locales, extranjeros o incluso virtuales.

\section{3.- LA CREACIÓN DEL ESPACIO EUROPEO DE EDUCACIÓN SUPERIOR VIR-} TUAL

El Espacio Europeo de Educación Superior tiene sus raíces en el Tratado de Maastricht (1992). De un modo más específico, el EEES se inicia con la Declaración de la Sorbona de 1998, que destacó el papel de las Universidades en el desarrollo de la dimensión cultural europea y de la Europa del conocimiento, y se consolida y se amplía con las Declaraciones de Bolonia (Junio de 1999), de Praga (2001) y de Berlín (Septiembre de 2003) y Bergen (Mayo de 2005). En ellas los Ministros europeos, acuerdan promover y desarrollar en sus respectivos países la reforma de la estructura y la organización de las enseñanzas universitarias para estimular la construcción de un Espacio Europeo de Educación Superior.

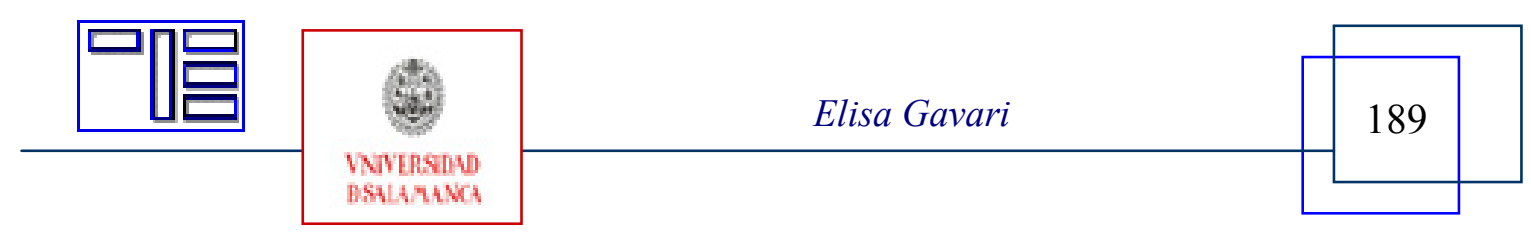


Revista Electrónica Teoría de la Educación.

Educación y Cultura en la Sociedad de la Información.

http://www.usal.es/teoriaeducacion

Vol. 7. No2. Diciembre 2006

La Declaración de Bolonia, firmada en el año 1999 y conducente a la creación del Espacio Europeo de Enseñanza Superior no menciona la importancia de la dimensión virtual (Dumort, 2002, 294). No será sino hasta el año 2000 cuando la Comisión Europea manifieste, por vez primera, la conciencia sobre el importante rol social y pedagógico actual de las NN.TT., a través de la iniciativa política e-Europe (Comisión Europea, 2000). Esta iniciativa está destinada a la potenciación de la aplicación de las TICs en todos los ámbitos de la sociedad europea y, en lo que al ámbito universitario se refiere, a la creación en última instancia de un Espacio Virtual Europeo de Enseñanza Superior (Dumort, 2002, 295).

La iniciativa Eeurope, y en particular, a través del programa E-learning (2004-2006) pretende la integración efectiva de las tecnologías de la información y la comunicación (TIC) en los sistemas de educación y formación en Europa. Dicho programa gira en torno a las siguientes líneas de acción: fomento de la alfabetización digital; los campus europeos virtuales; el hermanamiento electrónico de centros de enseñanza europeos y el fomento de la formación del profesorado; y, las acciones transversales para la promoción del e-learning en Europa. La segunda línea de acción Campus virtuales europeos pretende desarrollar los siguientes aspectos:

a) Desarrollo de los instrumentos existentes, en particular de aquellos relativos a la movilidad virtual, como complemento y refuerzo de la movilidad física (Erasmus virtual), a los sistemas de reconocimiento y validación (basados en los ECTS) y a los servicios de información y orientación, o cualquier otra sinergia entre modelos virtuales y tradicionales. Estos proyectos deberían basarse en acuerdos institucionales que, siempre que fuera posible, amplíen o complementen acuerdos de cooperación ya existentes en el marco de los programas comunitarios de movilidad.

b) Campus virtuales transnacionales. El programa apoyará proyectos estratégicos propuestos por centros de educación superior de, al menos, tres Estados miembros. Tendrían que elaborarse modelos de cooperación para el aprendizaje electrónico referentes a: el desarrollo de planes de estudio conjuntos por parte de varias universidades, incluidos acuerdos de evaluación, convalidación y reconocimiento de las competencias adquiridas, sujetos a los procedimientos nacionales; experimentos de movilidad virtual a gran escala como complemento de la movilidad física; desarrollo de planes duales de estudios innovadores, que combinen métodos de aprendizaje tradicionales y en línea.

c) Modelos europeos de aprendizaje electrónico para la educación superior. Estos proyectos deben elaborar nuevos modelos europeos de cooperación entre los centros de educación superior que traten, en particular, de la oferta de formación continua y desarrollo profesional, y de la creación de servicios de apoyo al aprendizaje, así como de la formación de profesores, formadores y demás personal educativo en el uso pedagógico del aprendizaje electrónico; deben examinarse métodos que garanticen la calidad; desarrollo de una mejor comprensión del cambio organizativo y de los posible riesgos que supone la aplicación del

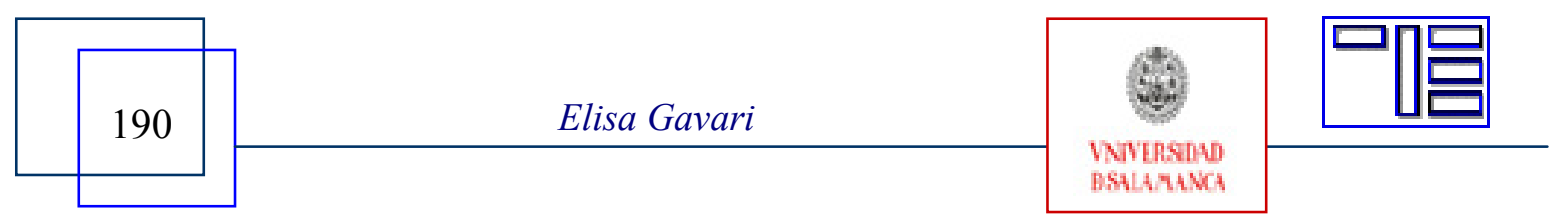


aprendizaje electrónico en la educación superior; elaboración de modelos europeos de asociación público-privada en el ámbito del aprendizaje electrónico en la educación superior, así como del aprovechamiento de las oportunidades que ofrecen los nuevos modelos de colaboración y financiación (http://www.ec.europa.eu/education/programmes/elearning/programme_es.html).

Para el logro efectivo de estos objetivos es necesario apoyarse sobre los principios que han guiado históricamente la enseñanza universitaria a distancia. En el siguiente apartado los vamos a revisar uno a uno.

\section{4.- LOS PRINCIPIOS RECTORES DE LA ENSEÑANZA SUPERIOR VIRTUAL}

Los principios rectores de la academia digital que ahora son redescubiertos por las autoridades europeas y que, poco a poco, se trasfieren a las universidades presenciales mediante las nuevas tecnologías se pueden sintetizar en tres: la igualdad de oportunidades y la calidad de la educación, el aprendizaje a lo largo de la vida, y el pensamiento pedagógico innovador. Estos principios han sido desarrollados por las universidades a distancia, desde su creación, y en la actualidad están siendo reclamados por la academia digital. En este apartado vamos a examinar las virtudes de los principios sobre los que se ha apoyado la enseñanza a distancia, que son los mismos sobre los que se debería apoyar la academia digital europea a través de las nuevas tecnologías.

\subsection{La igualdad de oportunidades}

Desde sus formas iniciales, empleando el correo postal, hasta las más sofisticadas formas digitales actuales (realidad que designamos como academia digital), la enseñanza a distancia ha desarrollado la dimensión compensatoria. A modo de ejemplo podemos acudir al artículo 3 de los Estatutos de la Open University, enmendados por el Privy Council en el año 2004, en el que se recoge que uno de los fines de la educación a distancia el "desarrollo y difusión del conocimiento y el aprendizaje mediante la enseñanza y la investigación a través de una diversidad de medios (...) y la provisión de educación de nivel profesional y universitario para sus estudiantes y la promoción del bienestar educativo de toda la comunidad". Más concreta es la página web de esta institución que, a fecha de abril de 2006, establece como su misión "la promoción de la igualdad de oportunidades y la justicia social mediante la provisión de enseñanza universitaria de calidad a todos los que deseen lograr sus ambiciones y actualizar su potencial" (http://www.open.ac.uk/about/ou/p2.shtml).

Ciertamente, las características de la enseñanza a distancia (muy particularmente, la inexistencia de numerus clausus, etc.) han contribuido a la universalización de la enseñanza superior. Esto está imponiendo a las instituciones de este nivel educativo dos retos: la ampliación del acceso a los servicios educativos, y la necesidad de atender a una población de estudiantes cada vez más diversificada que antaño y con una realidad presupuestaria limitada (Dumort, 2002, 290). El empleo de la tecnología educativa en la

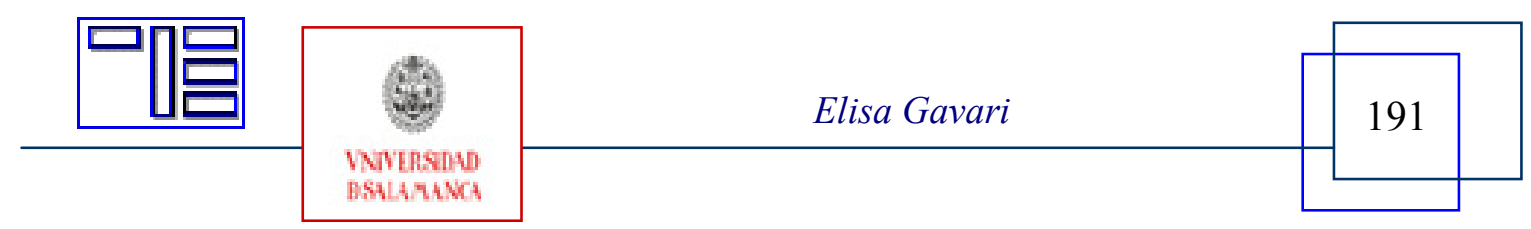


Revista Electrónica Teoría de la Educación.

Educación y Cultura en la Sociedad de la Información.

http://www.usal.es/teoriaeducacion

Vol. 7. No2. Diciembre 2006

enseñanza superior, se configura, paulatinamente como la solución ciertamente idónea para abordar de manera conjunta y acertada estos nuevos retos.

\subsection{El aprendizaje a lo largo de la vida}

El aprendizaje a lo largo de la vida (ALV) constituye el segundo de los principios rectores de la academia digital y, ciertamente, es una de las columnas vertebrales de la enseñanza a distancia desde sus orígenes. Así, el art. 4 de los nuevos Estatutos de la UNED, al especificar las funciones de la UNED en su servicio a la sociedad explicita como una de las más fundamentales "el establecimiento y desarrollo de programas de educación permanente, promoción cultural, perfeccionamiento y actualización profesional".

El paradigma del ALV, cuyos orígenes se remontan a la década de los sesenta, contiene concepciones y elementos pedagógicos que han sido contemplados y materializados por la modalidad de enseñanza a distancia desde sus inicios: la necesidad de la democratización incondicional del saber, el empleo eficiente de los recursos económicos, la necesidad de vincular la educación con la cultura que se desarrolla fuera de los muros institucionales y con el mundo laboral, la necesidad de modificar las estrategias de enseñanza y aprendizaje para desarrollar procesos educativos flexibles que permitan una mayor autonomía en el proceso de aprendizaje, etc.

Según autores como Jones, acabamos de inaugurar la "era del ALV, 1990-2030" (Jones, $1995,151)$. Esta realidad está siendo a la par secundada y promovida tanto por las diversas instancias internacionales (Unión Europea, OCDE, UNESCO) como, de forma unánime, por las políticas educativas de los países de la órbita occidental. La OCDE, a modo de ejemplo, organizó su reunión anual de ministros de educación en el año 1996 bajo el título de "Aprendizaje a lo largo de la vida para todos" (Field, 2000, 9). Esta agenda internacional y nacional de indiscutido consenso ha sancionado -sin pretenderlo explícitamente- la validez de la modalidad de la academia virtual, toda vez que, de forma característica, la promoción del ALV suele indefectiblemente vincularse en dichas agendas a su potenciación de forma mediatizada por las nuevas tecnologías.

\subsection{El pensamiento pedagógico innovador.}

El pensamiento y las prácticas pedagógicas innovadoras constituyen el tercer principio articulador de la academia digital. Este es un elemento manifiesto y evidente en esta modalidad de la enseñanza, y que se reconoce explícitamente en los estatutos de estas universidades: "Son funciones específicas de la UNED (...) incorporar, desarrollar y facilitar el uso de los métodos y sistemas tecnológicos que en cada momento mejor se adecúen al modelo educativo de la UNED" (BOE, 2005: art. 4). "La UNED imparte la enseñanza mediante la modalidad de educación a distancia, que se caracteriza por la utilización de una metodología didáctica específica con el empleo conjunto de medios impresos, audiovisuales y de las nuevas tecnologías (...) (idem: art. 8). La actualización del diseño de los métodos de enseñanza y aprendizaje y la promoción e incentivación de

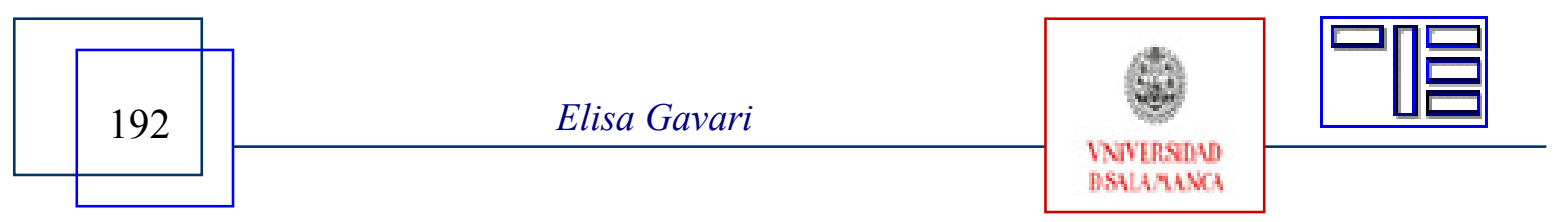


la edición del material didáctico en soporte informático u otro es encargado a unidades específicas como la Comisión de Metodología y Docencia de la UNED.

Particularmente, creemos que la concepción, el diseño y el desarrollo de la educación a distancia y la academia digital deriva de un pensamiento pedagógico innovador. La naturaleza y el carácter de nuestros estudiantes y de los procesos pedagógicos que se desarrollan en nuestras instituciones: sistema de libre acceso, muchas de cuyas opciones de estudio carecen de requisitos de ingreso; modularidad y flexibilidad de materiales de estudio; flexibilidad en lugar y tiempo de estudio; flexibilidad en el ritmo de estudio; pensamiento pedagógico centrado en el alumno que concibe a los alumnos como desempeñando un rol activo. Todas estas variables necesariamente reconocen implícitamente nuestra creencia en el autoaprendizaje, en el aprendizaje autónomo, en la capacidad de nuestros estudiantes de organizar su propio aprendizaje en términos de secuencia y también en términos de información y conocimiento. La misma Unión Europea vincula de forma inexorable las pedagogías innovadoras a las prácticas de enseñanza desarrolladas mediante las NN.TT. Así, en sus prioridades de actuación de una estrategia en apoyo del aprendizaje permanente, subraya especialmente: "potenciar la búsqueda de pedagogías innovadoras para los profesores, formadores y mediadores, teniendo en cuenta (...) la importancia cada vez mayor de las tecnologías de la información y la comunicación" (UE, 2001).

Ciertamente, la enseñanza personalizada no figura como pensamiento pedagógico explícitamente consagrado en los estatutos de la UNED. Pero, de lo que no cabe duda, es que en la modalidad de enseñanza a distancia a los alumnos se les exige un rol más autónomo y activo que a los alumnos de la enseñanza presencial y de que, dada la extraordinaria variedad de procedencia social de los alumnos, los profesores consagrados a la enseñanza a distancia somos especialmente proclives a valorar "la capacidad, interés, ritmo y circunstancias de cada estudiante" (Soacha, 2001), especialmente de aquellos con necesidades específicas. Se trata de un pensamiento pedagógico centrado en el alumno (Dumort, 2002, 292) que está muy en consonancia con la especificidad del rol y las funciones de la universidad a distancia. Cara al futuro, y desde la implantación plena de la academia digital, el reto más acuciante del pensamiento pedagógico innovador, qué duda cabe, es el que Evans y Nation han denominado como el "texto abierto", por el que "los estudiantes deben convertirse en colaboradores en el desarrollo de sus propios cursos mediante reflexión crítica" (vid. Evans and Nation, 1996). Se trata, en suma, de un nuevo paradigma académico que está siendo creado por el e-aprendizaje, que consagra un nuevo rol de los estudiantes en la creación del conocimiento y que tendrá implicaciones decisivas en cuestiones como comprensión del currículo y la certificación del conocimiento.

No olvidemos que la arquitectura del EEES de aprendizaje permanente se sostiene sobre tres pilares: una nueva estructura de títulos en dos niveles (grado y postgrado), una nueva forma de acreditar las competencias adquiridas por los alumnos en su proceso formativo (suplemento europeo al título) y el crédito ECTS o crédito europeo (cambios en la

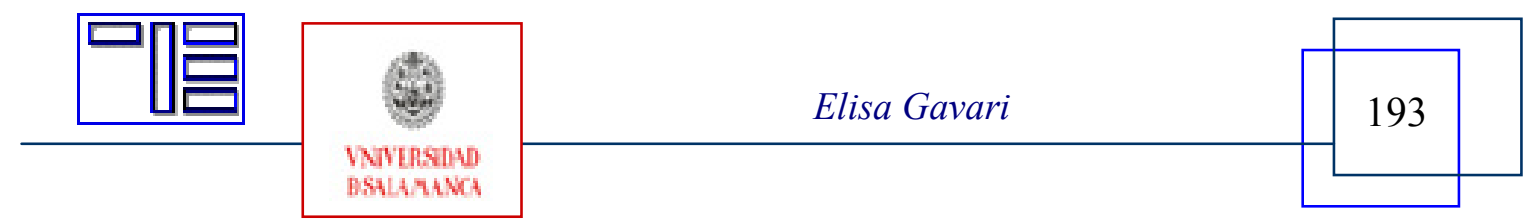


Revista Electrónica Teoría de la Educación.

Educación y Cultura en la Sociedad de la Información.

http://www.usal.es/teoriaeducacion

Vol. 7. No2. Diciembre 2006

metodología de la enseñanza y de aprendizaje). La adopción del crédito europeo hará posible y facilitará el reconocimiento académico completo y "a priori" de los estudios realizados en cualquier país de la Unión Europea, la movilidad estudiantil, el desarrollo de un currículo homologable internacionalmente, y la transferencia y comparabilidad de los aprendizajes de una institución a otra. Pero, sobre todo, significa la introducción de una nueva cultura de enseñanza-aprendizaje. Los principios de los ECTS son los siguientes:

- los ECTS se basan en el principio de que 60 créditos miden el volumen de trabajo de un estudiante a tiempo completo a lo largo de un curso académico;

- los créditos en términos de ECTS solamente se pueden obtener una vez que se ha realizado el trabajo y una adecuada valoración de los trabajos. Los resultados del aprendizaje se definen en términos de competencias que expresan lo que el estudiante sabrá, comprenderá y será capaz de hacer una vez que haya completado el proceso de aprendizaje.

- el trabajo del estudiante, medido en ECTS, se refiere al tiempo que se exige al alumno para completar todas las actividades planeadas como la asistencia a clases, seminarios, estudios privados, preparación de proyectos, exámenes, etc.

- los créditos se asignan a todos los elementos del programa de estudios (tales como módulos, cursos, prácticas, trabajo de disertación, etc.) y reflejan la cantidad de trabajo que hay que realizar.

La adopción de los créditos en la sociedad del conocimiento supone que los roles del profesor y del alumno están en un proceso de cambio. En la actualidad, los profesores deben adquirir las siguientes competencias:

- el dominio de procesos que determinan la generación, apropiación y uso del conocimiento;

- la capacidad de incorporar las nuevas tecnologías de información y comunicación al aprendizaje;

- los deseos de propiciar y facilitar el aprendizaje;

- la capacidad para despertar el interés, la motivación y el gusto por aprender;

- la disponibilidad para aprender por cuenta propia y a través de la interacción con otros;

- las habilidades para estimular la curiosidad, la creatividad y el análisis;

- las aptitudes para fomentar la comunicación interpersonal y el trabajo en equipo;

- la imaginación para identificar y aprovechar oportunidades diversas de aprendizaje;

- y, la autoridad moral.

En el nuevo milenio con la adopción de nuevos currículos escolares y universitarios, que se articulan alrededor de los créditos y de la noción de competencia, se pretende que el alumno universitario se convierta en un aprendiz autónomo y desarrolle competencias tales como: aprender a pensar; aprender a cooperar; aprender a comunicar sus conocimientos y sus opiniones teniendo claras las diferencias entre ambas; aprender a ser críti-

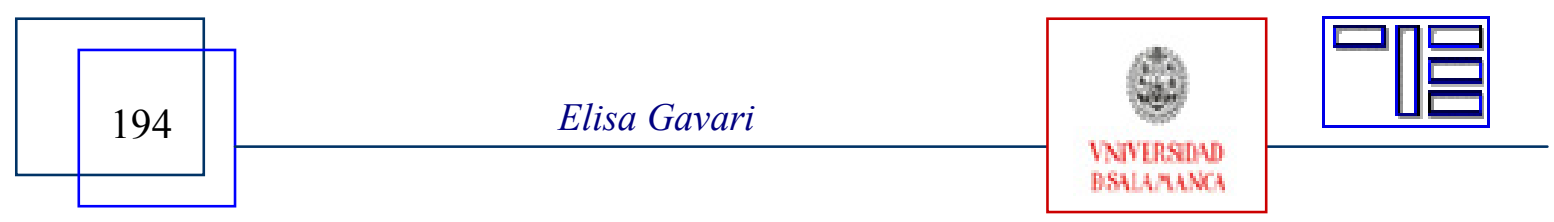


co frente al conocimiento, analizando los argumentos que lo apoyan y evaluando y sopesando la importancia de los datos y de las evidencias, de forma que permitan emitir juicios razonados y tomar decisiones consecuentes; y, a aprender a automotivarse, de forma que los alumnos sean capaces de establecer y gestionar sus metas y motivos.

\section{5.- LA MOVILIDAD VIRTUAL}

Hasta ahora cuando nos referíamos a la movilidad estudiantil en Europa nos referíamos siempre a la movilidad física de profesores y alumnos, en el marco del Programa ERASMUS y otros similares, basada en los créditos ECTS. La incorporación de las nuevas tecnologías masiva en Europa va a permitir la realización del ERASMUS de profesores y alumnos en la dimensión virtual. Una de las mayores virtudes de esta nueva movilidad va a ser la reducción de costes y tiempo. Esto va a permitir que algunas personas que no podían beneficiarse del Programa ERASMUS, por motivos económicos, ahora lo van a poder hacer. No obstante recordemos que este programa, recientemente galardonado, se considera la acción educativa más satisfactoria hasta el momento dentro de la política educativa europea. El desplazamiento a otro lugar, y sobre todo las experiencias reales vividas en otro entorno geográfico contribuye al enriquecimiento personal y a una mayor capacidad para la comprensión de la idiosincrasia de nuestros vecinos europeos.

\section{6.- CONCLUSIONES}

La construcción del Espacio Europeo de Educación Superior virtual requiere la aplicación de los principios que, desde antaño han regido la educación en las universidades a distancia, en universidades presenciales. Las nuevas tecnologías se revelan como el instrumento más adecuado que posibilita y optimiza la coexistencia de dichos principios. La asunción por parte de administradores, profesores y alumnos de las nuevas tecnologías no es automática sino que exige una gran inversión por adaptar los ECTS a las necesidades de profesores y alumnos. Así mismo la nueva metodología de enseñanza y aprendizaje que suponen tanto la participación en el Espacio Europeo de Educación Superior así como la introducción de las nuevas tecnologías supone un cambio en los roles de profesores y alumnos. Los profesores no son exclusivamente meros transmisores de contenidos sino de nuevas metodologías de aprendizaje, que guíen al alumno para que se convierta en un aprendiz autónomo y en constante reciclaje. Solamente así puede realizarse la creación de un Espacio Europeo de Educación Superior en sus dos dimensiones (presencial y virtual) que respondan al paradigma de la educación y formación a lo largo de la vida.

\section{7.- BIBLIOGRAFÍA}

BOE (21 de abril 2001): Real Decreto 408/2001, de 20 de abril, por el que se establece el II Plan de la Calidad de las Universidades.

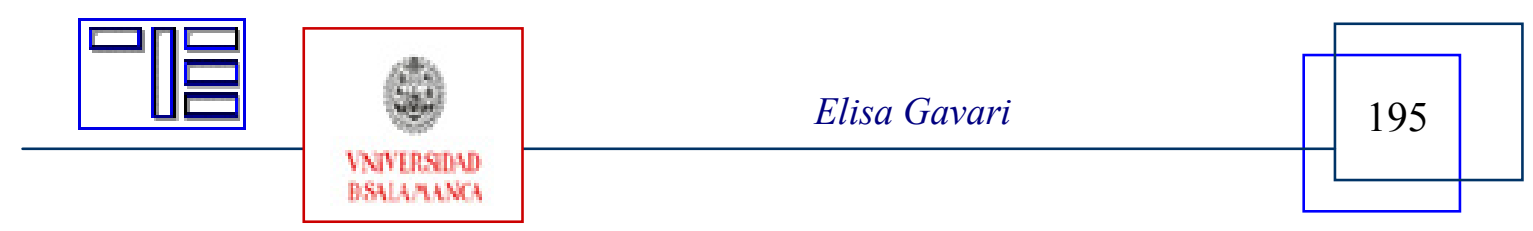


Revista Electrónica Teoría de la Educación.

Educación y Cultura en la Sociedad de la Información.

http://www.usal.es/teoriaeducacion

Vol. 7. No2. Diciembre 2006

BOE (16 de abril 2005): Real Decreto 426/2005, de 15 de abril, por el que se aprueban los Estatutos de la Universidad Nacional de Educación a Distancia.

COMMISSION OF THE EUROPEAN COMMUNITIES (2005): Digital Divide Forum Report: Broadband Access and Public Support in Under-Served Areas. Brussels.

DUMORT, A. (2002): "New media and distance education. EU and US perspectives". In DUTTON, W. H. and LOADER, B. D. (eds): Digital Academe. The New Media and Institutions of Higher Education and Learning. London, Routledge.

DUTTON, W. H. and LOADER, B. D. (eds): Digital Academe. The New Media and Institutions of Higher Education and Learning. London, Routledge.

EUROPEAN ASSOCIATION FOR QUALITY ASSURANCE IN HIGHER EDUCATION (2005): Standards and guidelines for Quality Assurance in the European Higher Education Area. Helsinki.

EVANS, T. and NATION, D. (eds) (1996): Opening Education. Policies and Practices from Open and Distance Education. London, Routledge.

MEC (1995): 'Proyecto de Ley Orgánica de Educación', Madrid.

FIELD, J. (2000): Lifelong Learning and the new educational order. Stoke on Trent, Trentham Books.

GARCÍA GARRIDO, J.L. and GARCÍA RUIZ, M.J. (2005): Temas candentes de la educación en el siglo XXI. Madrid, Ediasa.

GARCÍA HOZ, V. (1970): Educación personalizada. Madrid, Miñón.

JONES, B. (1995): Sleepers, Wake! Technology and the Future of Work. Melbourne, Oxford University Press.

MUSGRAVE, P. W. (1979): The Sociology of Education. London, Methuen. Third Edition.

NOLL, A. M. (2002): "Technology and the future of the university. A sober view". In DUTTON, W. H. and LOADER, B. D. (eds): Digital Academe. The New Media and Institutions of Higher Education and Learning. London, Routledge.

PERRATON, H. and LENTELL, H. (eds): Policy for open and distance learning. London, Routledge/Falmer.

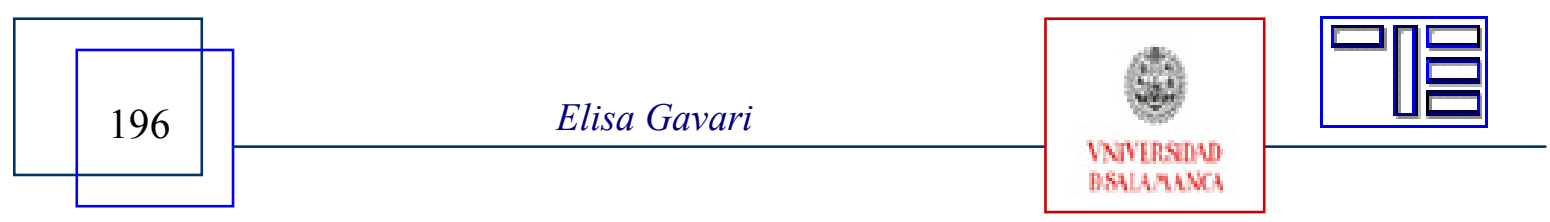


ROBINSON, B. (2004): "Governance, accreditation and quality assurance in open and distance education". In PERRATON, H. and LENTELL, H. (eds): Policy for open and distance learning. London, Routledge/Falmer.

SOACHA ORTEGA, J. H. (2001): “Origen de la educación personalizada”, Sappiens.

UE (2001): Comunicación de la Comisión de 21 de noviembre de 2001 - Hacer realidad un espacio europeo del aprendizaje permanente [COM (2001) 678 final].

UNED (2001): Informe Final de Evaluación de la Titulación de Geografía e Historia. Plan Nacional de Evaluación de la Calidad de las Universidades. Madrid.

UNED (2004): Reglamento de estudios de educación permanente de la Universidad Nacional de Educación a Distancia. Madrid.

\section{Para citar este artículo puede utilizar la siguiente referencia:}

GAVARI, Elisa. (2006): Los principios rectores del espacio europeo de educación superior virtual. En GARCÍA CARRASCO, Joaquín (Coord.) Estudio de los comportamientos emocionales en la red [monográfico en línea]. Revista Electrónica Teoría de la Educación: Educación y Cultura en la Sociedad de la Información. Vol. 7, no 2. Universidad de Salamanca. [Fecha de consulta: dd/mm/aaaa].

$<\mathrm{http} / / / w w w . u s a l . e s / \sim$ teoriaeducacion/rev_numero_07_02/n7_02_elisa_gavari.pdf $>$ ISSN 1138-9737

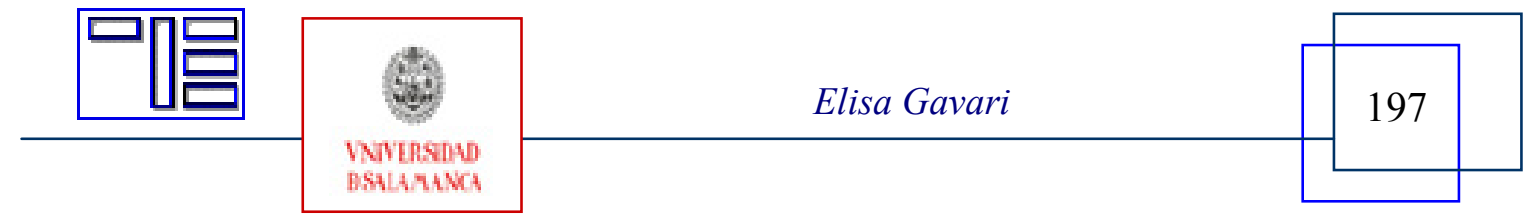

